AperTO - Archivio Istituzionale Open Access dell'Università di Torino

\title{
The JEM-EUSO mission: An introduction
}

\section{This is the author's manuscript}

Original Citation:

Availability:

This version is available http://hdl.handle.net/2318/1578033

since 2016-06-30T14:12:48Z

Published version:

DOI:10.1007/s10686-015-9482-x

Terms of use:

Open Access

Anyone can freely access the full text of works made available as "Open Access". Works made available under a Creative Commons license can be used according to the terms and conditions of said license. Use of all other works requires consent of the right holder (author or publisher) if not exempted from copyright protection by the applicable law. 


\section{ResearchGate}

See discussions, stats, and author profiles for this publication at:

https://www.researchgate.net/publication/283766405

\section{The JEM-EUSO mission: An introduction}

Article in Experimental Astronomy · November 2015

Impact Factor: 1.99 · DOI: 10.1007/s10686-015-9482-x

READS

361

288 authors, including:

\section{Alessandro Bruno}

Università degli Studi di Bari...

150 PUBLICATIONS 2,943

CITATIONS

SEE PROFILE

Luis Del Peral

University of Geneva

211 PUBLICATIONS 2,453

CITATIONS

SEE PROFILE 


\title{
The JEM-EUSO mission: An introduction
}

\author{
The JEM-EUSO Collaboration
}

Received: 14 August 2015 / Accepted: 19 October 2015 / Published online: 13 November 2015

(C) Springer Science+Business Media Dordrecht 2015

\begin{abstract}
The Extreme Universe Space Observatory on board the Japanese Experiment Module of the International Space Station, JEM-EUSO, is being designed to search from space ultra-high energy cosmic rays. These are charged particles with energies from a few $10^{19} \mathrm{eV}$ to beyond $10^{20} \mathrm{eV}$, at the very end of the known cosmic ray energy spectrum. JEM-EUSO will also search for extreme energy neutrinos, photons, and exotic particles, providing a unique opportunity to explore largely unknown phenomena in our Universe. The mission, principally based on a wide field of view (60 degrees) near-UV telescope with a diameter of $\sim 2.5 \mathrm{~m}$, will monitor the earth's atmosphere at night, pioneering the observation from space of the ultraviolet tracks (290-430 nm) associated with giant extensive air showers produced by ultra-high energy primaries propagating in the earth's atmosphere. Observing from an orbital
\end{abstract}

The JEM-EUSO Collaboration

The full author list and affiliations are given at the end of paper.

A. Santangelo

andrea.santangelo@uni-tuebingen.de

P. Picozza

Piergiorgio.Picozza@roma2.infn.it

T. Ebisuzaki

ebisu@postman.riken.jp 
altitude of $\sim 400 \mathrm{~km}$, the mission is expected to reach an instantaneous geometrical aperture of $A_{\text {geo }} \geq 2 \times 10^{5} \mathrm{~km}^{2}$ sr with an estimated duty cycle of $\sim 20 \%$. Such a geometrical aperture allows unprecedented exposures, significantly larger than can be obtained with ground-based experiments. In this paper we briefly review the history of space-based search for ultra-high energy cosmic rays. We then introduce the special issue of Experimental Astronomy devoted to the various aspects of such a challenging enterprise. We also summarise the activities of the on-going JEM-EUSO program.

Keywords Ultra-high energy cosmic rays $\cdot$ Neutrinos

\section{Introduction and history}

JEM-EUSO, the Extreme Universe Space Observatory on board the Japanese Experiment Module (JEM) of the International Space Station (ISS), is a pioneer mission designed to observe the most energetic particles in our universe, the ultra-high energy (UHE) cosmic rays with energies from a few $E \sim 10^{19} \mathrm{eV}$ to well beyond the threshold of the Greisen-Zatsepin-Kuzmin effect and up to the energy decade above $E \sim 10^{20} \mathrm{eV}$ [1], [2], [3].

The idea of space-based observations of UHE cosmic rays was first proposed by John Linsley in the late 70s, in response to a NASA Call for Projects and Ideas in High Energy Astrophysics for the 1980s (Fig. 1, [4]). The Satellite Observatory of Cosmic Ray Showers, SOCRAS, was indeed included in the final NASA Field Committee Report. The SOCRAS concept was very clear: to observe, by means of space-based devices looking to the nadir during night, the fluorescence light produced by giant extensive air showers (EAS) in the earth's atmosphere. SOCRAS was based on a $38 \mathrm{~m}$ diameter mirror to monitor a circular field of about $100 \mathrm{~km}$

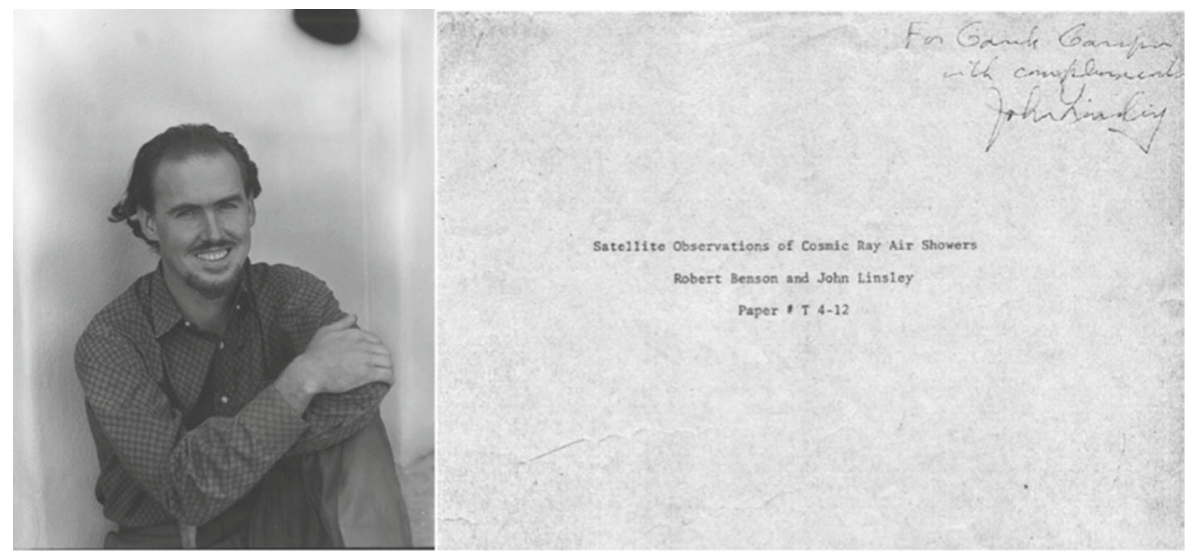

Fig. 1 Left: John Linsley at the Volcano Ranch times. Right: Cover page of the preprint in which Benson and Linsley (1981) presented SOCRAS [4] 
in diameter, corresponding to an area of $10^{4} \mathrm{~km}^{2}$ and an air mass of $\sim 10^{11}$ tons, from a circular orbit at $\sim 500-600 \mathrm{~km}$ above earth [6]. The idea, presented to the community at the 17th ICRC in Paris in 1981 by Benson and Linsley, was certainly visionary but unfortunately not feasible with the imaging and space technology of the 80s. In 1995 Linsley's original idea was rediscovered by Yoshiyuki Takahashi, who developed the concept of MASS, the Maximum-energy Auger (Air)-Shower Satellite. The key breakthrough in the imaging technology was the use of lightweight, unphased, segmented, double Fresnel lens optics to enlarge the field of view to about 30 degrees while keeping the telescope to a reasonable size [7]. In May 1995, Takahashi contacted John Linsley to discuss the new, now feasible, mission for UHE cosmic rays.

In the early 90s Linsley had moved to the Istituto di Fisica Cosmica con applicazioni dell'Informatica of the Italian National Research Council in Palermo, to work on the PLASTEX experiment with his old friend Livio Scarsi, and with Osvaldo Catalano. When John Linsley informed Livio Scarsi about the MASS idea, Scarsi, who was a prominent space scientist, heir of Giuseppe Occhialini, and who had worked with Linsley in Volcano Ranch, simply commented "It sounds as if might be fun" and suggested to change the name of MASS to something more general, something "easier to be explained to the space agencies: Airwatch", short for "Space Watch". The MASS/Airwatch concept was discussed in a seminal workshop in Huntsville in early August 1995 and later that month Takahashi presented the new idea at the 24th ICRC in Rome [7]. MASS was designed to image the earth's night sky on a high or low-orbit satellite at an altitude of $500-2000 \mathrm{~km}$, and was designed to accommodate fast CCDs or a large cluster of Multi Anode PMTs in the focal surface (Fig. 2).

The MASS idea evolved in 1996, in the US, into the Orbiting Wide Angle Light Concentrator (OWL), while the first Airwatch symposium was organised in Europe,

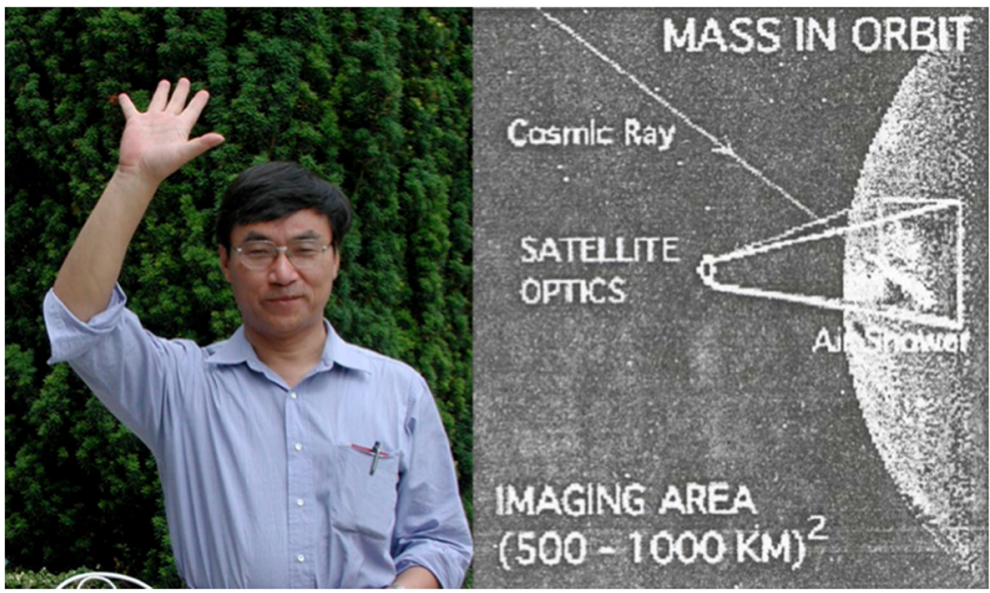

Fig. 2 Left: Yoshiyuki Takahashi on the occasion of the 7th Paris Cosmology Colloquium in 2002; Right: Cover of the original note on MASS, the Maximum-Energy Auger (Air)-Shower Satellite 
in Catania (Italy), in 1996. The OWL mission study proposal was accepted by NASA in 1996 and entered into NASA's Structure and Evolution of the Universe Mid-Term strategic plan in 2010. The OWL mission concept consists of two satellites observing in stereo configuration from an initial orbit at $\sim 1000 \mathrm{~km}$ that will reduce to $\sim 550 \mathrm{~km}$ at the end of the mission. The baseline OWL-eye instrument is a large f/1 Schmidt camera with a 45-degree full field of view and a $3.0 \mathrm{~m}$ entrance aperture. The entrance aperture is filled with a Schmidt corrector. The deployable primary mirror is $7 \mathrm{~m}$ in diameter. The focal plane has an area of $4 \mathrm{~m}^{2}$ segmented into about 1,300 multianode photomultiplier tubes for approximately 500,000 pixels. However, the mission has not yet been developed.

The Airwatch concept evolved in Europe into EUSO, the Extreme Universe Space Observatory, that Livio Scarsi first proposed as a free-flyer to the ESA's F2/F3 call in 2000. ESA selected the mission but re-oriented it as a payload for the Columbus module of the ISS [8], [9]. The phase-A study for the feasibility of EUSO, started in 2001 (see Fig. 3), was successfully completed in March 2004. Although EUSO was found technically ready to proceed into phase B, ESA did not continue the program mainly because of financial constraints in ESA and Europe, and because of the programmatic uncertainties of the ISS related to the Columbia accident.

In 2006, the Japanese and US teams, under the leadership of Yoshiyuki Takahashi, redefined the mission as an observatory attached to KIBO, the Japanese Experiment Module (JEM) of the ISS. They renamed the mission JEM-EUSO and started a new phase-A study targeting launch in 2013 in the framework of the second utilisation phase of the JEM/EF [1]. The kick-off meeting of the renewed EUSO mission was

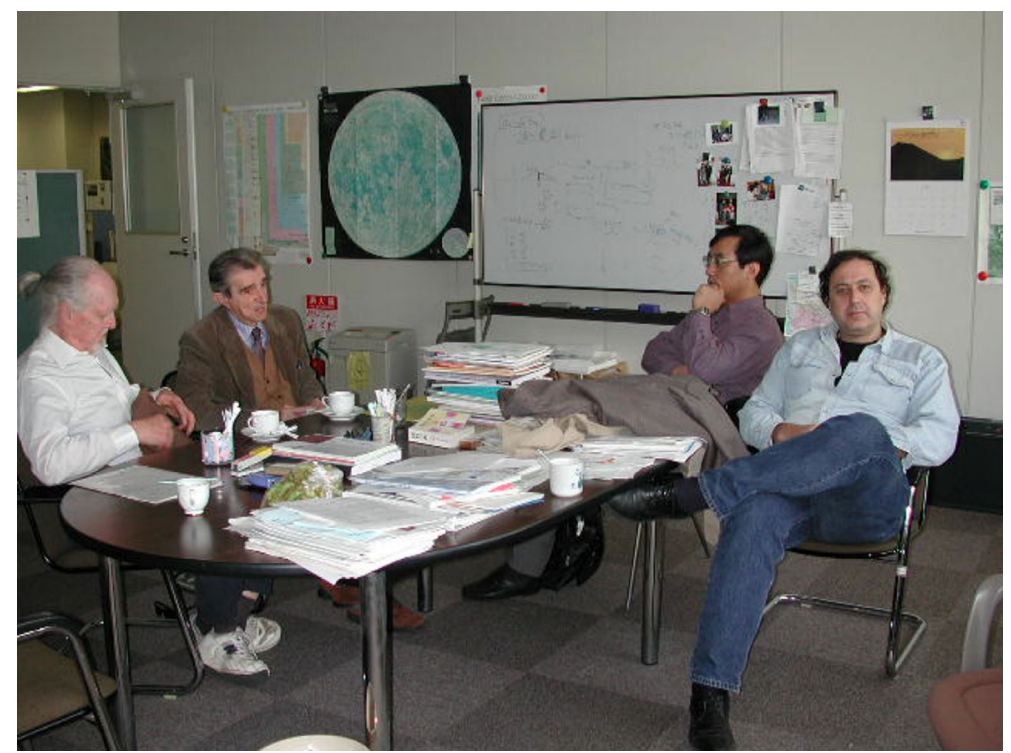

Fig. 3 From the left: John Linsley, Livio Scarsi, Yoshiyuki Takahashi, and Osvaldo Catalano at the Computational Astrophysics Laboratory on the occasion of an EUSO meeting in RIKEN, 2001 
held in RIKEN in 2006. In 2010 the EUSO mission was also included in the European Life and Physical Sciences in Space Programme (ELIPS) of ESA.

The Phase A/B1 study of JEM-EUSO led by JAXA continued with extensive simulations, design, and prototype developments, that significantly improved the JEM-EUSO mission profile, targeting eventually a launch in 2016 [10], [11], [12], [13].

This special issue of Experimental Astronomy comprises a series of papers which summarize all these efforts.

The current baseline of the JEM-EUSO instrument is described in [29]. In addition to the main UV telescope, an essential element of the JEM-EUSO payload is the Atmospheric Monitoring system, consisting of a LIDAR and an Infrared Camera. These are described in [18], [32] and [33]. According to the JEM-EUSO baseline, the life-time of the mission is five years. In the first two years the instrument points toward nadir direction, while for the remaining three years JEM-EUSO is planned to observe in tilted mode, that is with the telescope axis forming an angle with respect to nadir. The mission can be extended beyond the five years. The mission profile is summarised in Fig. 4.

According to the JAXA study, the JEM-EUSO instrument shall be transferred to the ISS by the HTV (H2 transfer vehicle). To accommodate JEM-EUSO into

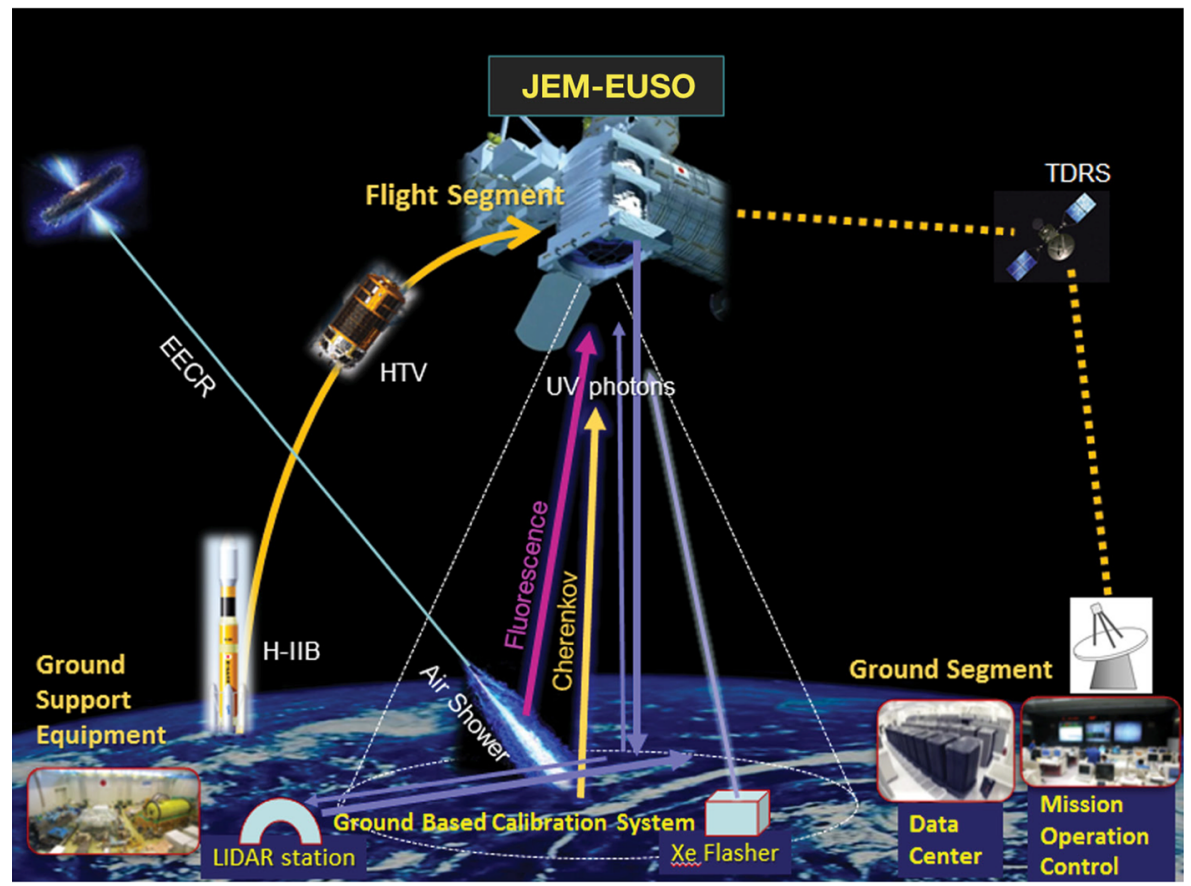

Fig. 4 The mission profile of the baseline JEM-EUSO mission as in the phase A/B1 of JAXA. JEMEUSO measures the fluorescence light produced by the extended air showers induced by UHE cosmic rays. Part of the signal is due to the scattered Cherenkov light and to the diffusively reflected Cherenkov light originating where the shower reaches the ground or the top of an optically thick cloud 
the volume of the HTV transfer vehicle, a contractible/extendable structure has been adopted. After the HTV docks in the ISS Docking Port, the Space Station Remote Manipulator System (SSRMS) takes out JEM-EUSO and passes it to the JEM Remote Manipulator System (JEMRMS). JEM-EUSO shall be attached to the Exposed Facility Unit \#2 of the JEM External Facility and then expanded to the operational configuration using the deploying mechanism. The principal components of the Ground Segment are the ISS ground station, the JEM Mission Control Room (MCR) in Tsukuba and the JEM-EUSO Science Data Centre (SDC). The end-to-end communication is established via NASA's Tracking and Data Relay Satellite (TDRS). The ground based calibration facility, equipped with Xenon flashers and lasers, is also an essential element of the ground segment.

Recently, a new study based on the Space-X Falcon 9 launching rocket and using Dragon as the transfer vehicle has been performed.

\section{Why a space-based mission to study UHE cosmic rays?}

The requirements, the expected performance, and the main features of JEM-EUSO are summarized in [3], [12], [14], [15], [16], [17]. The observational technique and exposure of JEM-EUSO is described in [23].

The most relevant advantage of space-based observations of UHE cosmic rays is the extremely large area that can be monitored from space. The instantaneous observational area is $\sim 2 \times 10^{5} \mathrm{~km}^{2}$ in nadir mode, implying a target air mass of more than $\sim 10^{12}$ ton, and can reach $\sim 7 \times 10^{5} \mathrm{~km}^{2}$ when the telescope axis is tilted with respect to nadir. These figures are almost two orders of magnitude larger than those of the largest ground based observatories, which amounts to $\sim 3 \times 10^{3} \mathrm{~km}^{2}$ for the Pierre Auger Observatory (Fig. 5).

A second relevant feature of the space-based approach to the observation of UHECRs is the highly uniform exposure over the full sky. JEM-EUSO, and UHECRs space observatories in general, naturally provide a $4 \pi$ sky coverage, in contrast to ground-based observatories that can observe only the southern or northern Hemispheres. The highly uniform exposure of JEM-EUSO, shown in Fig. 6, is essential to minimise systematics in the statistical analysis studies of arrival directions, needed to understand the anisotropy of UHECRs at various scales [25].

Another advantage is the large and well constrained distance between the instrument and the location of the extensive air shower (EAS). EASs are in fact constrained to a track length of $\sim 10-20 \mathrm{~km}$, rather small compared with the height $(\sim 400 \mathrm{~km})$ of the ISS orbit. In addition, space-based observatories have the possibility of observing in cloudy conditions since, in most cases, the maximum of the shower occurs above the cloud-top [24].

Assuming a duty cycle of $\sim 20 \%$, the currently expected trigger efficiencies, and an operation time of about five years, JEM-EUSO can reach an annual exposure close to an order of magnitude larger than the currently operating ground-based observatories. More details can be read in [3], [23]. 


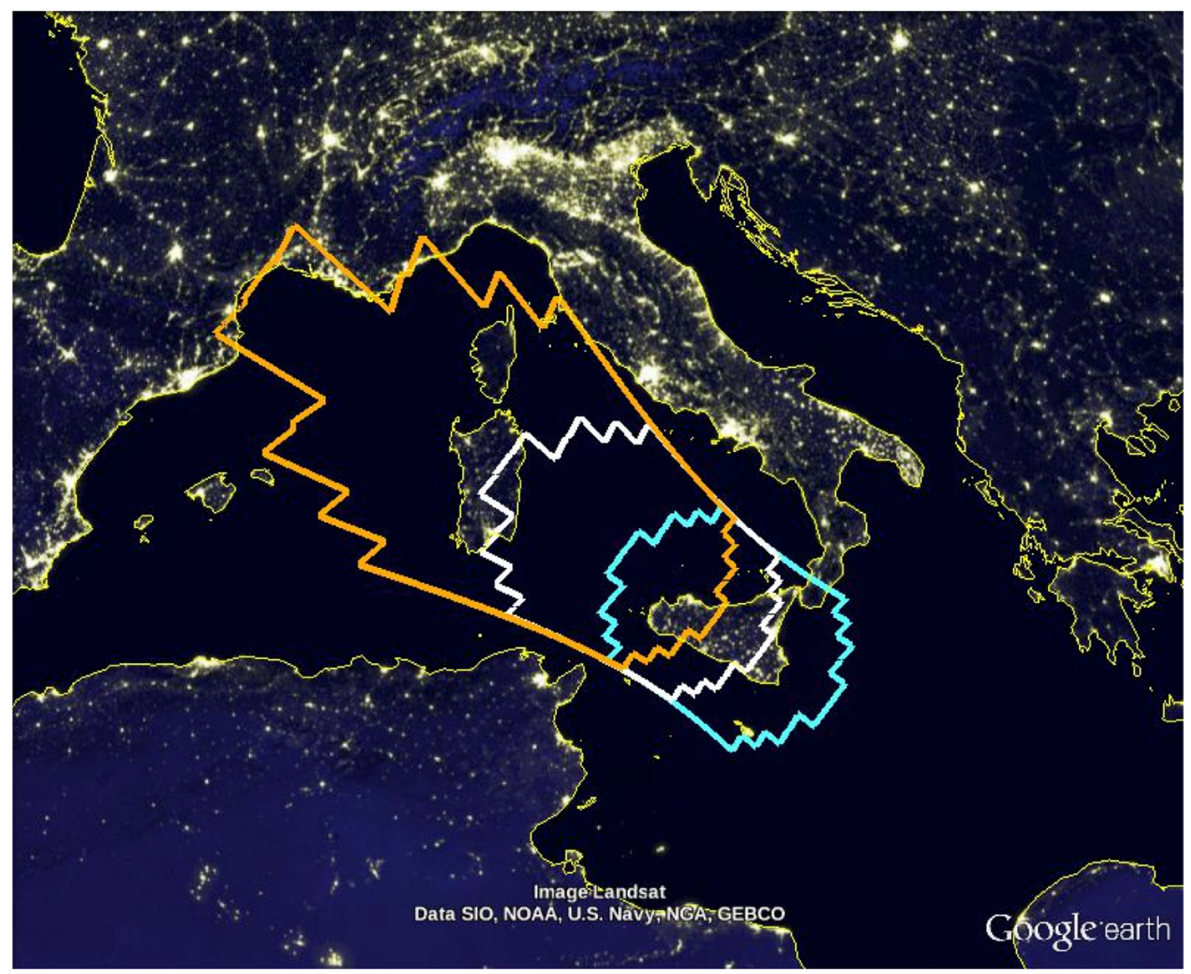

Fig. 5 Footprint of the field of view of the JEM-EUSO telescope projected above Sicily. It was at the Istituto di Fisica Cosmica con applicazioni dell'informatica of the italian Consiglio delle Ricerche, in Palermo, Sicily, that Livio Scarsi and John Linsley, with the significant contribution of Osvaldo Catalano, developed the EUSO mission concept. The blue profile corresponds to the field of view observed in nadir mode. The white and yellow curves refer to the field of view covered when JEM-EUSO is tilted by an angle of 20 and 30 degrees, respectively. The peculiar shape of the field of view is due to the shape of the optics that has been designed to be accommodated in the unpressurised module of the HTV, the Japanese transport vehicle to the ISS

\section{The JEM-EUSO pathfinders}

In parallel with the development of the main mission concept, the JEM-EUSO program has been enlarged to include a series of "pathfinders" (which are experiments to test the observational technique, and to validate the specific technologie).

\subsection{The EUSO-Balloon}

The EUSO-Balloon has been developed by the JEM-EUSO collaboration as a demonstrator for the specific technologies and methods featured in the main instrument. The mission was proposed by the French laboratories involved in JEM-EUSO and is led by the balloon division of the CNES, the French Space Agency. The instrument has been built by the JEM-EUSO collaboration. EUSO-Balloon is an imaging 

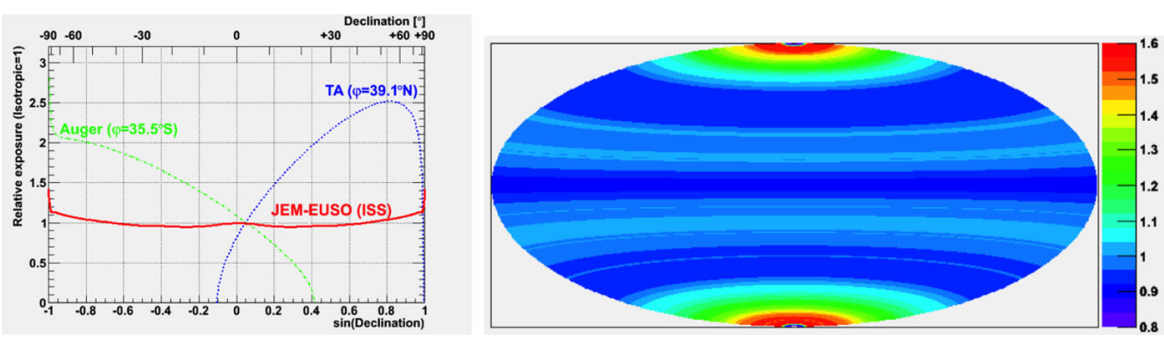

Fig. 6 Left: The exposure of the JEM-EUSO mission is almost uniform across the northern and southern hemispheres of the sky because of the inclination (51.6 degree) of the ISS. Right: the expected exposure map of the JEM-EUSO mission

UV telescope, a scaled version of the EUSO telescope, pointing towards the nadir from a float altitude of $\sim 40 \mathrm{~km}$. Using Fresnel Optics and a Photo-Detector Module, a prototype of the ones designed for the main mission, the instrument monitors a $12^{\circ} \times 12^{\circ}$ wide field of view in the wavelength range between 290 and $430 \mathrm{~nm}$, at a rate of 400,000 frames/s [26].

The first flight was launched on August 25, 2014, from the Timmins Stratospheric Balloon Base in Canada, in a CNES balloon campaign [27] (Fig. 7). The objectives of the EUSO-Balloon program are threefold: a) perform a full end-to-end test of a JEM-EUSO prototype consisting of all the main subsystems of the space experiment; b) image the UV background originating from the earth's surface, with spatial and temporal resolution relevant for JEM-EUSO; c) detect the tracks of ultraviolet light due to UHE cosmic rays for the first time from near space. The first flight was indeed very successful. The background was measured under several conditions and although no cosmic ray tracks were detected, the instrument was able to detect artificial UV tracks induced by a laser beam shot from a helicopter flying in the field of view of the balloon. The main features of the instrument and mission, together with several results of the first flight, are summarised in [27]. Given the success of the first flight, the EUSO-Balloon program will continue with future flights. An opportunity to fly over the ocean from Aire sur 1Adour, France, with an improved instrument is under consideration for 2016. The next major step of the balloon program will be a long duration flight with a NASA Super Pressure Balloon, that will allow the first observations of UHECRs from near space, and the test of potentially viable, new technologies such as SiPMs for the focal surface.

\subsection{The EUSO-TA}

EUSO-TA, where TA stands for Telescope Array, is a ground-based testing campaign of a downscaled prototype of the JEM-EUSO telescope, developed by the JEMEUSO Consortium in collaboration with the Institute for Cosmic Ray Research, of the University of Tokyo, and the Telescope Array collaboration. A fully functional 


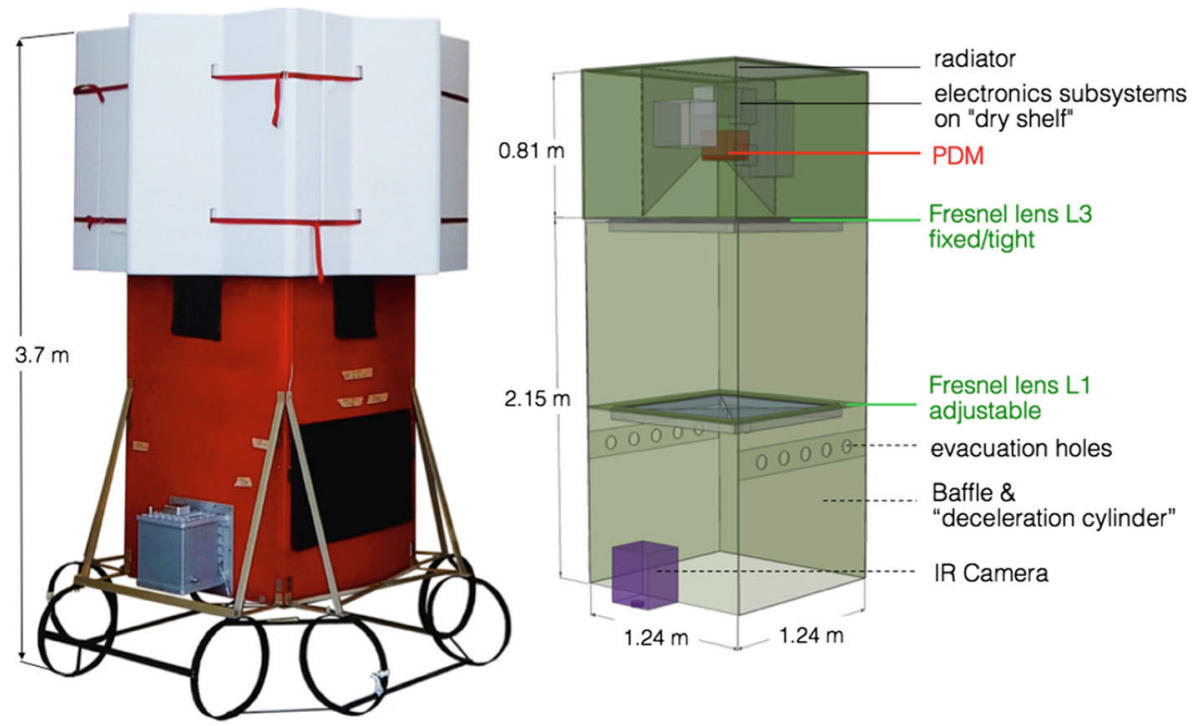

Fig. 7 Left: schematic view of the instrument booth of the EUSO-Balloon. Right: schematic view of the optical bench. The configuration is the one for the first flight launched from the Timmins base in August 2014.

prototype of JEM-EUSO has been built and installed at Black Rock Mesa, Utah, at the site of the Telescope Array UHECR observatory [36]. EUSO-TA will observe artificial light produced by the electron light source and the central laser facility of the TA calibration system. EUSO-TA is also designed to observe tracks induced by cosmic rays, simultaneously with TA, that provides the external trigger. This allows a deeper understanding of the EUSO response and systematics. EUSO-TA will also perform studies of the transverse profile of the shower with spatial resolution better than that of the TA fluorescence detector (TA-FD). A description of the main features and goals of EUSO-TA can be found in [28] (Fig. 8).

EUSO-TA is currently taking data in a series of rather successful measurement campaigns. At the time of writing, EUSO-TA has already properly detected artificial EAS tracks simulated with the portable laser system from the Colorado Mines school and has detected its first cosmic ray events.

\section{What comes next?}

Unfortunately the JEM-EUSO mission in its baseline configuration, as designed in the phase A/B1 study, has been frozen by JAXA due to the restructuring of the space station program of Japan. In addition, the HTV launch program will most likely be reduced. 


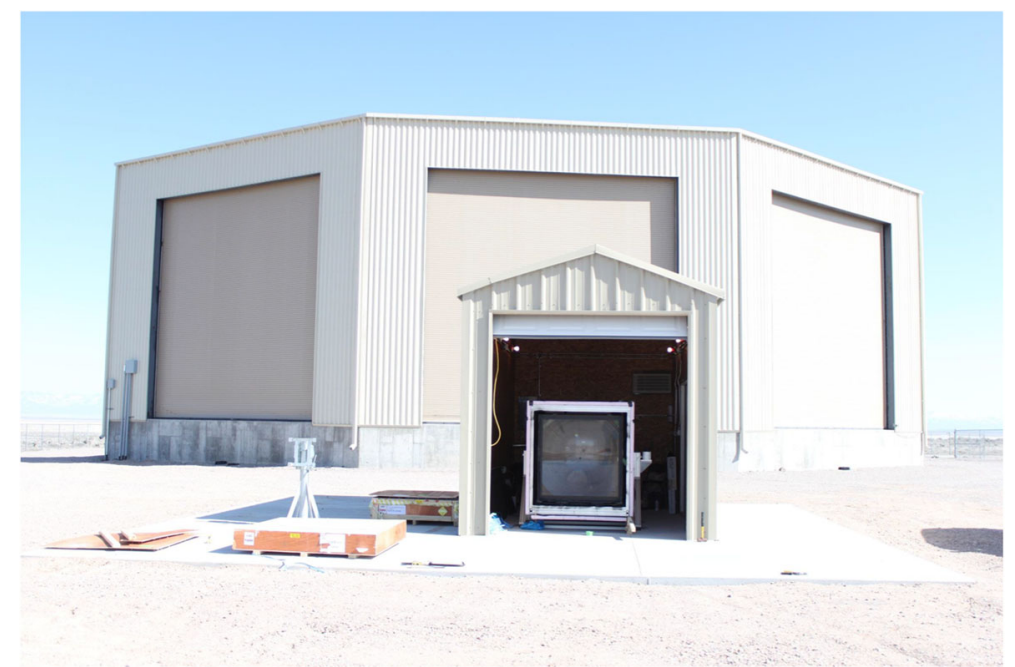

Fig. 8 EUSO-TA installed at the TA-FD station in Black Rock Mesa, Utah

The US team is currently pursuing, with the support of the collaboration, the goal of reorienting the mission using the Falcon 9 launcher and the Dragon transport vehicle to accommodate the mission on the JEM module. The so-called "Dragon" option also impacts the design of the instrument since a circular optics and focal surface can now be used, instead of the side-cut design needed for HTV. Preliminary simulations show that the JEM-EUSO performances summarised in the special issue of Experimental Astronomy can be reached with the new configuration, and in some cases improved.

A different parallel approach is also being actively studied by the JEM-EUSO collaboration: an improved version of the Russian KLYPVE mission, defined as KLYPVE-EUSO or K-EUSO for short. The KLYPVE project, already included into the ROSCOSMOS long term program of experiments on board the Russian segment of the ISS, uses a compound mirror concentrator instead of Fresnel lenses, reaching a better efficiency but a smaller field of view. Major improvements of the KLYPVEEUSO mission are the use of a Fresnel corrector lens to significantly reduce the size of the reflected spot on the focal surface, and new elements of the structure and electronics. The mission is planned to be launched in 2020. More details can be found in [19].

The JEM-EUSO collaboration is also developing and actually building a new pathfinder mission: Mini-EUSO. Mini-EUSO, already included in the ISS science programs of ROSCOSMOS and the Italian Space Agency (ASI), is a small, compact UV telescope to be inside the Russian Module of the ISS. It will measure the UV background from earth. Mini-EUSO will be placed in the nadir looking UV window in the Russian segment of the ISS. In addition to measuring and monitoring the UV emission of night-time earth, Mini-EUSO will study UV atmospheric and bioluminescence phenomena. It will also observe several meteors. Launch is foreseen in 2017. 


\section{The special issue of experimental astronomy on JEM-EUSO}

The special issue on JEM-EUSO summarises many of the efforts of the JEM-EUSO collaboration to develop the science case as well as the experimental, technological, and engineering aspects of such a challenging pioneer mission. The expected performance, obtained by careful end to end simulations, are also an important contribution to the special issue.

The science aspects of the exploratory objectives, UHE photons and neutrinos are discussed in [20], while the science of the atmospheric phenomena is presented in [21], with a focus on meteors and exotic nuclearites in [22]. Two papers are devoted to the JEM-EUSO observation technique, also discussing observations in cloud conditions ([23], [24]). The instrument is summarised in [29], while details on the photo-detector module are presented in [31] and its calibration in [30]. The other key element of the JEM-EUSO instrument, the AM system, is presented in [32], while the details of the IR camera are discussed in [33]. The angular and energy resolution ( obtained with end to end simulations) are discussed in [34] and [35]. Finally, the pathfinders, including the TUS mission onboard the Lomonosov satellite, are presented in [27], [28] and [37].

The JEM-EUSO collaboration includes, as of today, 16 Countries $^{1}$, 80 Institutions, and more than 300 researchers.

It is always difficult to predict the future. We do not know exactly how or when this mission will be launched. Hopefully it will be launched in the next few years. There are no doubts that the corpus of the papers included in this issue constitutes an invaluable base for any future studies in the field.

This special issue is dedicated to the memory of John Linsley, Livio Scarsi, and Yoshi Takahashi, whose relentless efforts and creative, contagious enthusiasm opened the field of space-base exploration of the Ultra High Energy Universe.

Acknowledgments The work on JEM-EUSO and its pathfinders has been supported by the Basic Science Interdisciplinary Research Projects of RIKEN and JSPS KAKENHI Grant (22340063, 23340081, and 24244042), by the Italian Ministry of Foreign Affairs, General Direction for the Cultural Promotion and Cooperation, by the Deutsches Zentrum für Luft- und Raumfahrt, by the Helmholtz Alliance for Astroparticle Physics HAP funded by the Initiative and Networking Fund of the Helmholtz Association (Germany), and by Slovak Academy of Sciences MVTS JEM-EUSO as well as VEGA Grant agency Project 2/0076/13. The Spanish Consortium involved in the JEM-EUSO Space Mission is funded by MICINN under Projects AYA200906037-E/ESP, AYA-ESP 2010 19082, AYA201129489-C0301, AYA201239115-C03 01, CSD200900064 (Consolider MULTIDARK) and by Comunidad de Madrid (CAM) under Project S2009/ ESP-1496. The EUSO Balloon has been supported by the French CNES and IN2P3. The US is supported by the NASA grants NNX13AH55G, NNX13AH53G. Russia is supported by the Russian Foundation for Basic Research Grant No 13-02-12175-ofi-m. Moreover, studies for JEMEUSO have been partly funded by the European Space Agency (ESA) through the "EUSO" Topical Team Fund.

\footnotetext{
${ }^{1}$ Countries member of the JEM-EUSO collaboration are: Algeria Bulgaria, France, Germany, Italy, Japan,
} Korea, Mexico, Poland, Romania, Russia, Slovakia, Spain, Sweden, Switzerland and USA 


\section{References}

1. Takahashi, Y. et al.: The JEM-EUSO Mission. New. J. of Phys. 11, 065009 (2009)

2. Santangelo, A., Petrolini, A.: Observing ultra-high-energy cosmic particles from space: S-EUSO, the Super-Extreme Universe Space Observatory Mission. New J. of Phys. 11, 065010 (2009)

3. Adams Jr., J.H. et al.: An evaluation of the exposure in nadir observation of the JEM-EUSO mission. Astropart. Phys. 44, 76-90 (2013)

4. Benson, R., Linsley, J.: Satellite observation of cosmic ray air showers. In: 17th Int. Cosmic Ray Conf. Proc., Paris, France, p. 8 (1981)

5. Linsley, J.: Proc. of the Workshop on Very High Energy Cosmic-Ray Interactions Uni- versity of Pennsylvania Cherry, M.L., Lande, K., Steinberg, R.I. (eds.), vol. 476 (1982)

6. Scarsi, L. et al.: EUSO - Extreme Universe Space Observatory. In: 27th Int. Cosmic Ray Conf. Proc., Hamburg, Germany, HE (2001)

7. Takahashi, Y.: Maximum-energy Auger Air Shower Satellite (MASS) for Observing Cosmic Rays in the Energy Region 10 ${ }^{19-22}$ eV. In: 24th Int. Cosmic Ray Conf. Proc., Rome, Italy, vol. 3 (1995)

8. Parmar, A., et al.: The Extreme Universe Space Observatory (EUSO) Mission in the Context of ESA. In: 28th Int.1 Cosmic Ray Conf. Proc. Tsukuba, Japan, 1073-1076 (2003)

9. Gianfiglio, G., Ostillio, A., Santangelo, A.: EUSO in the Context of ESA Human Spaceflight Directorate. In: 28th Int. Cosmic Ray Conf. Proc. Tsukuba, Japan, 1093-1096 (2003)

10. Takahashi, Y.: JEM EUSO mission on the International Space Station. J. Phys.: Conf. Ser. 120(6), 062013 (2008)

11. Ebisuzaki, T. et al.: The JEM-EUSO mission. In: 38th COSPAR Scientific Assembly, Bremen, Germany, Symposium E, session 18, paper number E18-0030-10 (2010)

12. Santangelo, A. et al.: Unveiling the UHE Universe from space: the JEM-EUSO mission. Nucl. Phys. B Proc. Suppl. 212, 368-378 (2011)

13. Ebisuzaki, T., et al.: The JEM-EUSO mission. In: 32nd Int. Cosmic Ray Conf. Proc., Beijing, China, Vol. 3 HE1.4 (2011)

14. Ebisuzaki, T., Median Tanco, G., Santangelo, A.: The JEM-EUSO mission. Adv. Space Res. 53(10) (2014)

15. Bertaina, M. et al.: Performance of JEM-EUSO, UHECR 2012 - International Symposium on Future Directions in UHECR Physics, CERN, Geneva, EPJ Web of Conferences, 53, id.09002 (2013)

16. Casolino, M., et al.: Detecting ultra-high energy cosmic rays from space with unprecedented acceptance: objectives and design of the JEM-EUSO mission. Astrophys. Space Sci. Trans 7(4) (2011)

17. bertaina, M., et al.: Performance and air-shower reconstruction techniques for the JEM-EUSO mission. Adv. Space Res. 53(10) (2014)

18. Morales de los Rios, J.A. et al.: for the JEM-EUSO Collaboration: The Infrared Camera Prototype Characterization for the JEM-EUSO Space Mission. NIMA 749, 74 (2014)

19. Panasyuk, M. et al.: The current status of orbital experiments for UHECR studies. In: Proceedings of the 24th European Cosmic Ray Symposium, Kiel, Germany. arXiv:1501.06368 (2015)

20. The JEM-EUSO collaboration: Ultra High Energy Photons and Neutrinos with JEM-EUSO, Experimental Astronomy, special issue on JEM-EUSO doi:10.1007/s10686-013-9353-2 (2014)

21. The JEM-EUSO collaboration: Science of atmospheric phenomena with JEM-EUSO, Experimental Astronomy, special issue on JEM-EUSO doi:10.1007/s10686-014-9431-0 (2015)

22. The JEM-EUSO collaboration: JEM-EUSO: Meteor and nuclearite observations, Experimental Astronomy, special issue on JEM-EUSO doi:10.1007/s10686-014-9375-4 (2014)

23. The JEM-EUSO Collaboration: JEM-EUSO observational technique and exposure, Experimental Astronomy doi:10.1007/s10686-014-9376-3 (2014)

24. The JEM-EUSO Collaboration: The JEM-EUSO observation in cloudy conditions, Experimental Astronomy doi:10.1007/s10686-014-9377-2 (2014)

25. Bertaina, M., Parizot, E.: for the JEM-EUSO collaboration: The JEM-EUSO mission: a space observatory to study the origin of Ultra-High Energy Cosmic Rays. Nucl. Phys. B (Proc Suppl) 256 (2014)

26. von Ballmoos, P. et al.: (The JEM-EUSO collaboration): A balloon-borne prototype for demonstrating the concept of JEM-EUSO. Adv. Space Res. 53, 1544 (2014)

27. The JEM-EUSO Collaboration: The eusoballoon pathfinder, Experimental Astronomy, special issue on JEM-EUSO doi:10.1007/s10686-015-9467-9 (2015) 
28. The JEM-EUSO collaboration: Ground-based tests of JEM-EUSO components at the Telescope Array site, "EUSO-TA", Experimental Astronomy, special issue on JEM-EUSO doi:10.1007/s10686-015-9441-6 (2015)

29. The JEM-EUSO Collaboration: The JEM-EUSO Instrument, Experimental Astronomy, special issue on JEM-EUSO doi:10.1007/s10686-014-9418-x (2015)

30. The JEM-EUSO Collaboration: The calibration aspects of the JEM-EUSO mision, Experimental Astronomy, special issue on JEM-EUSO doi:10.1007/s10686-015-9453-2 (2015)

31. The JEM-EUSO Collaboration: The Photodetector module of the JEM-EUSO mision, Experimental Astronomy, manuscript in preparation (2015)

32. The JEM-EUSO Collaboration: The AM system of the JEM-EUSO instrument, Experimental Astronomy, special issue on JEM-EUSO doi:10.1007/s10686-014-9431-0 (2014)

33. The JEM-EUSO Collaboration: The Infrared Camera onboard JEM-EUSO, Experimental Astronomy, special issue on JEM-EUSO doi:10.1007/s10686-014-9402-5 (2014)

34. The JEM-EUSO Collaboration: Performances of JEM-EUSO: angular resolution, Experimental Astronomy, special issue on JEM-EUSO doi:10.1007/s10686-013-9371-0 (2014)

35. The JEM-EUSO Collaboration: Performances of JEM-EUSO: energy and Xmax reconstruction, Experimental Astronomy, special issue on JEM-EUSO doi:10.1007/s10686-014-9427-9 (2015)

36. Kawai, H. et al.: Telescope array experiment. Nuclear Phys. B (Proc Suppl.), 175-176 (2008)

37. The JEM-EUSO collaboration: The Space Experiment TUS on Board the Lomonosov Satellite Pathfinder of the JEM-EUSO Space Experiment, Experimental Astronomy, special issue on JEMEUSO, submitted (2015)

\section{The JEM-EUSO Collaboration}

J.H. Adams Jr. ${ }^{m d}$, S. Ahmad ${ }^{b b}$, J.-N. Albert ${ }^{b a}$, D. Allard ${ }^{b c}$, L. Anchordoqui ${ }^{m f}$, V. Andreev ${ }^{m e}$, A. Anzalone ${ }^{d h, d n}$, Y. Arai ${ }^{e v}$, K. Asano ${ }^{e t}$, M. Ave Pernas ${ }^{k c}$, P. Baragatti ${ }^{d o}$, P. Barrillon ${ }^{b a}$, T. Batsch ${ }^{h c}$, $\mathrm{J}$ Bayer $^{c d}$, R. Bechini ${ }^{d l}$, T. Belenguer ${ }^{k b}$, R. Bellottid ${ }^{d a}, d b$, K. Belov ${ }^{m e}$, A.A. Berlind ${ }^{m h}$, M. Bertaina ${ }^{d k, d l}$, P.L. Biermann ${ }^{c b}$, S. Biktemerova ${ }^{i a}$, C. Blaksley ${ }^{b c}$, N. Blanc ${ }^{l a}$, J. Błęcki ${ }^{h d}$, S. Blin-Bondil ${ }^{b b}$, J. Blümer $^{c b}$, P. Bobik ${ }^{j a}$, M. Bogomilov ${ }^{a} a$, M. Bonamente ${ }^{m d}$, M.S. Briggs ${ }^{m d}$, S. Briz ${ }^{k d}$, A. Bruno ${ }^{d a}$, F. Cafagna ${ }^{d a}$,

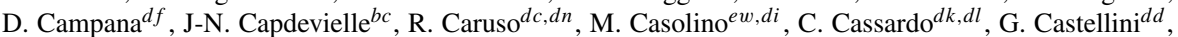
C. Catalano ${ }^{b d}$, O. Catalano ${ }^{d h, d n}$, A. Cellino ${ }^{d k, d m}$, M. Chikawa ${ }^{e d}$, M.J. Christl ${ }^{m g}$, D. Cline ${ }^{m e}$, V. Connaughton ${ }^{m d}$, L. Conti ${ }^{d o}$, G. Cordero ${ }^{g a}$, H.J. Crawford ${ }^{m a}$, R. Cremonini ${ }^{d l}$, S. Csorna ${ }^{m h}$, S. Dagoret-Campagne ${ }^{b a}$, A.J. de Castro ${ }^{k d}$, C. De Donato ${ }^{d i}$, C. de la Taille ${ }^{b b}$, C. De Santis ${ }^{d i, d j}$, L. del Peral ${ }^{k c}$, A. Dell'Oro ${ }^{d k, d m}$ N. De Simone ${ }^{d i}$, M. Di Martino ${ }^{d k, d m}$, G. Distratis ${ }^{c d}$, F. Dulucq ${ }^{b b}$, M. Dupieux ${ }^{b d}$, A. Ebersoldt ${ }^{c b}$, T. Ebisuzaki ${ }^{e w}$, R. Engel ${ }^{c b}$, S. Falk ${ }^{c b}$, K. Fang ${ }^{m b}$, F. Fenu ${ }^{c d}$, I. FernándezGómez $^{k d}$, S. Ferrarese ${ }^{d k, d l}$, D. Finco ${ }^{d o}$, M. Flamini ${ }^{d o}$, C. Fornaro ${ }^{d o}$, A. Franceschi ${ }^{d e}$, J. Fujimoto ${ }^{e v}$, M. Fukushima ${ }^{e g}$, P. Galeotti ${ }^{d k, d l}$, G. Garipov ${ }^{i c}$, J. Geary $^{m d}$, G. Gelmini ${ }^{m e}$, G. Giraudo ${ }^{d k}$, M. Gonchar ${ }^{i a}$, C. González Alvarado ${ }^{k b}$, P. Gorodetzky ${ }^{b c}$, F. Guarino ${ }^{d f, d g}$, A. Guzmán ${ }^{c d}$, Y. Hachisu ${ }^{e w}$, B. Harlov ${ }^{i b}$, A. Haungs ${ }^{c b}$, J. Hernández Carretero ${ }^{k c}$, K. Higashide ${ }^{e r, e w}$, D. Ikeda ${ }^{e g}$, H. Ikeda ${ }^{e p}$, N. Inoue ${ }^{e r}$, S. Inoue ${ }^{e g}$, A. Insolia ${ }^{d c, d n}$, F. Isgrò ${ }^{d f, d p}$, Y. Itow ${ }^{e n}$, E. Joven ${ }^{k e}$, E.G. Judd ${ }^{m a}$, A. Jung ${ }^{f b}$, F. Kajino ${ }^{e i}$, T. Kajino ${ }^{e l}$, I. Kaneko ${ }^{e w}$, Y. Karadzhov ${ }^{a a}$, J. Karczmarczyk ${ }^{h c}$, M. Karus ${ }^{c b}$, K. Katahira ${ }^{e w}$, K. Kawai ${ }^{e w}$, Y. Kawasaki ${ }^{e w}$, B. Keilhauer ${ }^{c b}$, B.A. Khrenov ${ }^{i c}$, Jeong-Sook Kim ${ }^{f a}$, Soon-Wook Kim ${ }^{f a}$, Sug$\mathrm{Whan} \mathrm{Kim}^{f d}$, M. Kleifges ${ }^{c b}$, P.A. Klimov ${ }^{i c}$, D. Kolev ${ }^{a a}$, I. Kreykenbohm ${ }^{c a}$, K. Kudela ${ }^{j a}$, Y. Kurihara ${ }^{e v}$, A. Kusenko ${ }^{m e}$, E. Kuznetsov ${ }^{m d}$, M. Lacombe ${ }^{b d}$, C. Lachaud ${ }^{b c}$, J. Lee ${ }^{f c}$, J. Licandro ${ }^{k e}$, H. Lim ${ }^{f c}$, F. López ${ }^{k d}$, M.C. Maccarone ${ }^{d h, d n}$, K. Mannheim ${ }^{c e}$, D. Maravilla ${ }^{g a}$, L. Marcelli ${ }^{d j}$, A. Marini ${ }^{d e}$, O. Martinez ${ }^{g c}$, G. Masciantonio ${ }^{d i, d j}$, K. Mase ${ }^{e a}$, R. Matev ${ }^{a a}$, G. Medina-Tanco ${ }^{g a}$, T. Mernik ${ }^{c d}$, H. Miyamoto $b a$, Y. Miyazaki ${ }^{e c}$, Y. Mizumoto ${ }^{e l}$, G. Modestino ${ }^{d e}$, A. Monaco ${ }^{d a}, d b$, D. MonnierRagaigne $^{b a}$, J.A. Morales de los Ríos ${ }^{k a, k c}$, C. Moretto ${ }^{b a}$, V.S. Morozenko ${ }^{i c}$, B. Mot ${ }^{b d}$, T. Murakami ${ }^{e f}$, M. Nagano ${ }^{e c}$, M. Nagata ${ }^{e h}$, S. Nagataki ${ }^{e k}$, T. Nakamura ${ }^{e j}$, T. Napolitano ${ }^{d e}$, D. Naumov ${ }^{i a}$, R. Nava ${ }^{g a}$, A. Neronov ${ }^{l b}$, K. Nomoto ${ }^{e u}$, T. Nonaka ${ }^{e g}$, T. Ogawa ${ }^{e w}$, S. Ogio ${ }^{e o}$, H. Ohmori ${ }^{e w}$, A.V. Olinto ${ }^{m b}$, P. Orleański ${ }^{h d}$, G. Osteria ${ }^{d f}$, M.I. Panasyuk ${ }^{i c}$, E. Parizot ${ }^{b c}$, I.H. Park ${ }^{f c}$, H.W. Park ${ }^{f c}$, B. Pastircak ${ }^{j a}$, T. Patzak ${ }^{b c}$, T. Paul ${ }^{m f}$, C. Pennypacker ${ }^{m a}$, S. Perez Cano ${ }^{k c}$, T. Peter ${ }^{l c}$, P. Picozza ${ }^{d i, d j, e w}$, T. Pierog $^{c b}$, L.W. Piotrowski ${ }^{e w}$, S. Piraino ${ }^{c d, d h}$, Z. Plebaniak ${ }^{h c}$, A. Pollini ${ }^{l a}$, P. Prat ${ }^{b c}$, G. Prévôt ${ }^{b c}$, H. Prieto $^{k c}$, M. Putis ${ }^{j a}$, P. Reardon ${ }^{m d}$, M. Reyes ${ }^{k e}$, M. Riccide, I. Rodríguez ${ }^{k d}$, M.D. Rodríguez Frías ${ }^{k c}$, 
F. Ronga ${ }^{d e}$, M. Roth ${ }^{c b}$, H. Rothkaehi ${ }^{h d}$, G. Roudil ${ }^{b d}$, I. Rusinov ${ }^{a a}$, M. Rybczyński ${ }^{h a}$, M.D. Sabau ${ }^{k b}$, G. Sáez Cano ${ }^{k c}$, H. Sagawa ${ }^{e g}$, A. Saito ${ }^{e j}$, N. Sakaki ${ }^{c b}$, M. Sakata ${ }^{e i}$, H. Salazar ${ }^{g c}$, S. Sánchez ${ }^{k d}$,

A. Santangelo ${ }^{c d}$, L. Santiago Crúz ${ }^{g a}$, M. Sanz Palomino ${ }^{k b}$, O. Saprykin ${ }^{i b}$, F. Sarazin ${ }^{m c}$, H. Sato ${ }^{e i}$, M. Sato ${ }^{e s}$, T. Schanz ${ }^{c d}$, H. Schieler ${ }^{c b}$, V. Scotti ${ }^{d f, d g}$, A. Segreto ${ }^{d h, d n}$, S. Selmane ${ }^{b c}$, D. Semikoz ${ }^{b c}$, M. Serra ${ }^{k e}$, S. Sharakin ${ }^{i c}$, T. Shibata ${ }^{e q}$, H.M. Shimizu ${ }^{e m}$, K. Shinozaki ${ }^{e w, c d}$, T. Shirahama ${ }^{e r}$, G. Siemieniec-Oziębło ${ }^{h b}$, H.H. Silva López ${ }^{g a}$, J. Sledd ${ }^{m g}$, K. Słomińska ${ }^{h d}$, A. Sobey ${ }^{m g}$, T. Sugiyama ${ }^{e m}$, D. Supanitsky ${ }^{g a}$, M. Suzuki ${ }^{e p}$, B. Szabelska ${ }^{h c}$, J. Szabelski ${ }^{h c}$, F. Tajima ${ }^{e e}$, N. Tajima ${ }^{e w}$, T. Tajima ${ }^{c c}$, Y. Takahashi ${ }^{e s}$, H. Takami ${ }^{e v}$, M. Takeda ${ }^{e g}$, Y. Takizawa ${ }^{e w}$, C. Tenzer ${ }^{c d}$, O. Tibolla ${ }^{c e}$, L. Tkachev ${ }^{i a}$,

H. Tokuno ${ }^{e t}$, T. Tomida ${ }^{e w}$, N. Tone ${ }^{e w}$, S. Toscano ${ }^{l b}$, F. Trillaud ${ }^{g a}$, R. Tsenov ${ }^{a a}$, Y. Tsunesada ${ }^{e t}$, K. Tsuno ${ }^{e w}$, T. Tymieniecka ${ }^{h c}$, Y. Uchihori ${ }^{e b}$, M. Unger ${ }^{c b}$, O. Vaduvescu ${ }^{k e}$, J.F. Valdés-Galicia ${ }^{g a}$, P. Vallania ${ }^{d k, d m}$, L. Valore ${ }^{d f, d g}$, G. Vankova ${ }^{a a}$, C. Vigorito ${ }^{d k, d l}$, L. Villaseñor ${ }^{g b}$, P. von Ballmoos ${ }^{b d}$, S. Wada ${ }^{e w}$, J. Watanabe ${ }^{e l}$, S. Watanabe ${ }^{e s}$, J. Watts Jr. ${ }^{m d}$, M. Weber ${ }^{c b}$, T.J. Weiler ${ }^{m h}$, T. Wibig ${ }^{h c}$, L. Wiencke ${ }^{m c}$, M. Wille ${ }^{c a}$, J. Wilms ${ }^{c a}$, Z. Włodarczyk ${ }^{h a}$, T. Yamamoto ${ }^{e i}$, Y. Yamamoto ${ }^{e i}$, J. Yang ${ }^{f b}$, H. Yano ${ }^{e p}$, I.V. Yashin ${ }^{i c}$, D. Yonetoku ${ }^{e f}$, K. Yoshida ${ }^{e i}$, S. Yoshida ${ }^{e a}$, R. Young ${ }^{m g}$, M.Yu. Zotov ${ }^{i c}$, A. Zuccaro Marchi ${ }^{e w}$

a St. Kliment Ohridski University of Sofia, Bulgaria

${ }^{b a}$ LAL, Univ Paris-Sud, CNRS/IN2P3, Orsay, France

${ }^{b b}$ Omega, Ecole Polytechnique, CNRS/IN2P3, Palaiseau, France

${ }^{b c}$ APC, Univ Paris Diderot, CNRS/IN2P3, CEA/Irfu, Obs. de Paris, Sorbonne Paris Cité, France

${ }^{b d}$ IRAP, Université de Toulouse, CNRS, Toulouse, France

ca ECAP, University of Erlangen-Nuremberg, Germany

${ }^{c b}$ Karlsruhe Institute of Technology (KIT), Germany

${ }^{c c}$ Ludwig Maximilian University, Munich, Germany

${ }^{c d}$ Inst. for Astronomy and Astrophysics, Kepler Center, University of Tübingen, Germany

ce Institut für Theoretische Physik und Astrophysik, University of Würzburg, Germany

da Istituto Nazionale di Fisica Nucleare - Sezione di Bari, Italy

${ }^{d b}$ Universita' degli Studi di Bari Aldo Moro and INFN - Sezione di Bari, Italy

$d c$ Dipartimento di Fisica e Astronomia - Universita' di Catania, Italy

${ }^{d d}$ Consiglio Nazionale delle Ricerche (CNR) - Ist. di Fisica Applicata Nello Carrara, Firenze, Italy

de Istituto Nazionale di Fisica Nucleare - Laboratori Nazionali di Frascati, Italy

$d f$ Istituto Nazionale di Fisica Nucleare - Sezione di Napoli, Italy

$d g$ Universita' di Napoli Federico II - Dipartimento di Scienze Fisiche, Italy

${ }^{d h}$ INAF - Istituto di Astrofisica Spaziale e Fisica Cosmica di Palermo, Italy

${ }^{d i}$ Istituto Nazionale di Fisica Nucleare - Sezione di Roma Tor Vergata, Italy

dj Universita' di Roma Tor Vergata - Dipartimento di Fisica, Roma, Italy

${ }^{d k}$ Istituto Nazionale di Fisica Nucleare - Sezione di Torino, Italy

${ }^{d l}$ Dipartimento di Fisica, Universita' di Torino, Italy

${ }^{d m}$ Osservatorio Astrofisico di Torino, Istituto Nazionale di Astrofisica, Italy

${ }^{d n}$ Istituto Nazionale di Fisica Nucleare - Sezione di Catania, Italy

do UTIU, Dipartimento di Ingegneria, Rome, Italy

$d p$ DIETI, Universita' degli Studi di Napoli Federico II, Napoli, Italy

ea Chiba University, Chiba, Japan

${ }^{e b}$ National Institute of Radiological Sciences, Chiba, Japan

${ }^{e c}$ Fukui University of Technology, Fukui, Japan

ed Kinki University, Higashi-Osaka, Japan

ee Hiroshima University, Hiroshima, Japan

ef Kanazawa University, Kanazawa, Japan

${ }^{e g}$ Institute for Cosmic Ray Research, University of Tokyo, Kashiwa, Japan

${ }^{e h}$ Kobe University, Kobe, Japan

ei Konan University, Kobe, Japan

ej Kyoto University, Kyoto, Japan

ek Yukawa Institute, Kyoto University, Kyoto, Japan

${ }^{e l}$ National Astronomical Observatory, Mitaka, Japan

em Nagoya University, Nagoya, Japan

en Solar-Terrestrial Environment Laboratory, Nagoya University, Nagoya, Japan

eo Graduate School of Science, Osaka City University, Japan 
${ }^{e p}$ Institute of Space and Astronautical Science/JAXA, Sagamihara, Japan

eq Aoyama Gakuin University, Sagamihara, Japan

er Saitama University, Saitama, Japan

${ }^{e s}$ Hokkaido University, Sapporo, Japan

${ }^{e t}$ Interactive Research Center of Science, Tokyo Institute of Technology, Tokyo, Japan

${ }^{e u}$ University of Tokyo, Tokyo, Japan

${ }^{e v}$ High Energy Accelerator Research Organization (KEK), Tsukuba, Japan

${ }^{e w}$ RIKEN, Wako, Japan

${ }^{f a}$ Korea Astronomy and Space Science Institute (KASI), Daejeon, Republic of Korea

${ }^{f b}$ Ewha Womans University, Seoul, Republic of Korea

$f^{f c}$ Sungkyunkwan University, Seoul, Republic of Korea

${ }^{f d}$ Center for Galaxy Evolution Research, Yonsei University, Seoul, Republic of Korea

${ }^{g a}$ Universidad Nacional Autónoma de México (UNAM), Mexico

${ }^{g b}$ Universidad Michoacana de San Nicolas de Hidalgo (UMSNH), Morelia, Mexico

${ }^{g c}$ Benemérita Universidad Autónoma de Puebla (BUAP), Mexico

ha Jan Kochanowski University, Institute of Physics, Kielce, Poland

$h b$ Jagiellonian University, Astronomical Observatory, Krakow, Poland

${ }^{h c}$ National Centre for Nuclear Research, Lodz, Poland

${ }^{h d}$ Space Research Centre of the Polish Academy of Sciences (CBK), Warsaw, Poland

ia Joint Institute for Nuclear Research, Dubna, Russia

${ }^{i b}$ Central Research Institute of Machine Building, TsNIIMash, Korolev, Russia

${ }^{i c}$ Skobeltsyn Institute of Nuclear Physics, Lomonosov Moscow State University, Russia

${ }^{j a}$ Institute of Experimental Physics, Kosice, Slovakia

${ }^{k a}$ Consejo Superior de Investigaciones Científicas (CSIC), Madrid, Spain

${ }^{k b}$ Instituto Nacional de Técnica Aeroespacial (INTA), Madrid, Spain

${ }^{k c}$ Universidad de Alcalá (UAH), Madrid, Spain

${ }^{k d}$ Universidad Carlos III de Madrid, Spain

${ }^{k e}$ Instituto de Astrofísica de Canarias (IAC), Tenerife, Spain

${ }^{l a}$ Swiss Center for Electronics and Microtechnology (CSEM), Neuchâtel, Switzerland

${ }^{l b}$ ISDC Data Centre for Astrophysics, Versoix, Switzerland

${ }^{l c}$ Institute for Atmospheric and Climate Science, ETH Zürich, Switzerland

${ }^{m a}$ Space Science Laboratory, University of California, Berkeley, USA

${ }^{m b}$ University of Chicago, USA

${ }^{m c}$ Colorado School of Mines, Golden, USA

${ }^{m d}$ University of Alabama in Huntsville, Huntsville, USA

me University of California (UCLA), Los Angeles, USA

${ }^{m f}$ University of Wisconsin-Milwaukee, Milwaukee, USA

$m g$ NASA - Marshall Space Flight Center, USA

${ }^{m h}$ Vanderbilt University, Nashville, USA 